\title{
Obtención, Evaluación y Congelación de Semen de Antilope Adulto (Addax nasomaculatus) del Zoo-Parque Lecocq de Montevideo-Uruguay
}

\author{
Collection, Evaluation and Freezing of Sperm Antilope Adult (Addax nasomaculatus) \\ of the Lecocq Zoo-Park in Montevideo-Uruguay
}

Calvo, J.*; Elhordoy, D.**; Modernel, A.***; Tavares, E.***; Aragunde, R.**;

Rohrer, V.***; Robert, D.*; Viqueira, M.* \& Lombide, P.*

CALVO, J.; ELHORDOY, D.; MODERNEL, A.; TAVARES, E.; ARAGUNDE, R.; ROHRER, V.; ROBERT, D.; VIQUEIRA, M. \& LOMBIDE, P. Obtención, evaluación y congelación de semen de antilope adulto (Addax nasomaculatus) del zoo-parque Lecocq de Montevideo-Uruguay. Int. J. Morphol., 34(1):276-279, 2016.

RESUMEN: La criopreservación de semen es una importante biotecnología reproductiva que viene siendo utilizada con éxito desde hace casi 50 años que busca promover la conservación del germoplasma masculino. Si bien en la actualidad se han desarrollado sistemas electrónicos automatizados portátiles, muchos técnicos continúan utilizando el método convencional de congelación por su practicidad, bajo costo y su buena eficiencia. En este trabajo, utilizando protocolos estandarizados para la evaluación reproductiva de machos de otras especies se presentan datos preliminares sobre obtención, evaluación y congelación de gameto masculino de antílopes adultos (Addax nasomaculatus). Se obtuvo el semen de tres antílopes machos adultos, registrándose volumen, color, motilidad, vigor, vitalidad, concentración y morfología. El semen fue congelado por el método convencional y preservado en nitrógeno a $-196{ }^{\circ} \mathrm{C}$. Los machos respondieron satisfactoriamente al método de sedación y extracción de semen. Tanto la evaluación del semen fresco como del semen congelado mostraron datos preliminares aceptables que validan la metodología utilizada y estimulan a continuar trabajando con esta especie dada su condición de animales en cautiverio y en peligro de extinción.

PALABRAS CLAVE: Semen; Criopreservación; Antílope.

\section{INTRODUCCIÓN}

La criopreservación de semen es una importante biotecnología reproductiva, que busca promover la conservación del germoplasma masculino por tiempo indeterminado. El objetivo de congelar células espermáticas es la producción de un banco de las mismas, teniendo a disposición un material sin plazo de vencimiento, potencializando la eficiencia de la reproducción animal (Amirat et al., 2004). Permite reducir costos, tales como el mantenimiento de animales, así como el control de la transmisión de enfermedades (de Souza Castelo et al., 2008). Sin embargo, el proceso de criopreservación resulta en la disminución de la fertilidad cuando se compara con semen fresco (Forero Gonzalez, 2004). La curva de enfriamiento utilizada durante la congelación tiene influencia directa en el grado de las lesiones celulares, debido a procesos de deshidratación y formación de cristales de hielo intracelulares (Moore et al., 2006). Si bien en la actualidad se han desarrollado sistemas electróni- cos automatizados portátiles, de bajo costo para el control de las curvas de enfriamiento y congelación del semen (Forero Gonzalez), muchos técnicos de campo utilizan el método convencional de congelación, utilizando materiales simples como la nevera de la propiedad, para realizar la curva de enfriamiento, y la congelación en la caja de poliestireno con nitrógeno líquido. Aunque la estandarización de las curvas de enfriamiento y congelación es imprecisa, ya que dependen de la calidad del material utilizado, del aislamiento de la nevera, del tamaño de la caja de poliestireno y el nivel de nitrógeno entre otras cosas, esta técnica ha demostrado ser viable y confiable. Sin embargo, el método convencional de congelación de semen continúa siendo una técnica de bajo costo, el semen o las pajuelas cargadas son refrigerados a $4{ }^{\circ} \mathrm{C}$, durante $4 \mathrm{~h}$. Luego de este periodo, se cargan las pajuelas y se colocan en una gradilla de metal, las que son transferidas para una caja de poliestireno, conteniendo ni-

* Área Histología y Embriología, Facultad de Veterinaria, Universidad de la República, Montevideo, Uruguay.

** Área Teriogenología, Facultad de Veterinaria, Universidad de la República, Montevideo, Uruguay.

*** Zoo-Parque Lecocq, Intendencia de Montevideo, Montevideo, Uruguay. 
trógeno líquido y acomodadas $6 \mathrm{~cm}$ por encima de la superficie liquida durante $20 \mathrm{~min}$. Finalmente, las pajuelas son inmersas en nitrógeno líquido y, posteriormente, almacenadas en un termo de nitrógeno. Esta biotecnología de congelación por el método tradicional de semen viene siendo utilizada con éxito desde hace casi 50 años. Los diferentes protocolos no solo procuran la obtención de altas tasas de sobrevivencia de las células, sino también la minimización de los efectos nocivos que la utilización de los crioprotectores produce sobre la viabilidad celular. Actualmente diferentes biotecnologías reproductivas están siendo utilizadas en especies silvestres, particularmente aquellas que se encuentran en peligro de extinción, siendo la congelación del gameto masculino una de ellas.

El Addax nasomaculatus es una de las especie de antílope adaptada a vivir en las regiones más áridas del continente africano. Se encuentra en la lista de especies amenazadas y en peligro de extinción por la Unión Internacional para la Conservación de la Naturaleza (UICN). Actualmente sólo menos de cien ejemplares se encuentran en estado libre, limitándose su presencia al parque nacional de Marruecos y algunos ejemplares en zoológico. En el Zoo-Parque Lecocq en Santiago Vázquez a 19 km al oeste de Montevideo en Uruguay se encuentra una población en cautiverio, considerada la más grande de Sudamérica; siendo la tercera en número de animales después de la de Texas y San Diego en Estados Unidos (Leizagoyen, 2005).

Las autoridades y profesionales veterinarios del ZooParque Lecocq vienen realizando esfuerzos para aumentar el número de ejemplares efectuando un manejo reproductivo que pueda dar variabilidad suficiente a la población de antílopes (Leizagoyen). Actualmente docentes de la Facultad de Veterinaria y veterinarios del Zoo-Parque Lecocq han establecido un marco de cooperación para la preservación de gametos de esta y otras especies en peligro de extinción. En este trabajo, utilizando protocolos estandarizados para la evaluación reproductiva de machos de otras especies de mamíferos se presentan datos preliminares sobre obtención, evaluación y congelación de gameto masculino de antílopes adultos (Addax nasomaculatus), una especie "En Peligro Crítico" de extinción (Lista Roja, IUCN - International Union for Conservation of Nature and Natural Resources) (Newby \& Wacher, 2008).

\section{MATERIAL Y MÉTODO}

Obtención del semen. En las instalaciones del Zoo-Parque Lecocq de Montevideo-Uruguay se obtuvo bajo sedación y mediante estimulación con electro eyaculador Pulsator IV
Lane M. Inc. ${ }^{\circledR}$ el semen de tres antílopes machos adultos siguiendo las normas de manejo bioético previsto en la legislación vigente en Uruguay. El semen obtenido fue diluido 1:1 en diluyente de semen bovino Minitube ${ }^{\circledR}$ refrigerado a $4^{\circ} \mathrm{C}$ y trasladado al laboratorio de Histología y Embriología de la Facultad de Veterinaria (45 min aproximadamente) para su evaluación y posterior congelación.

Análisis Espermático (Espermiograma). Los eyaculados fueron evaluados según el procedimiento descrito por el Departamento de Reproducción Animal, Facultad de Veterinaria (Uruguay) el año 2000 y Januskauskas \& Zilinskas (2002) para otras especies. Se registro volumen, color, motilidad progresiva (porcentaje de espermatozoides con movimiento de avance progresivo y uniforme), así como su vigor (intensidad del movimiento), utilizándose una escala subjetiva escala de 0 a 5, donde 0: sin movimiento, 5: fuerte movimiento de avance), vitalidad espermática, concentración y morfología espermática.

La motilidad y vigor se evaluaron a partir de una alícuota de semen depositada sobre una lámina y cubierta con cubreobjetos. La observación se realizo en microscopio óptico Olympus CX21 equipado con platina térmica Tokai Hit y con el objetivo de 100X. La motilidad se caracterizó por la presencia de movimientos rectilíneos uniformes y fue expresada en porcentaje ( 0 a $100 \%$ ). El vigor fue caracterizado de acuerdo con la intensidad de los movimientos espermáticos según una puntuación de 0 a 5. La concentración espermática fue evaluada luego de la dilución de 1:20 del semen en una solución formol-salina al $10 \%$. Una cámara de Neubauer fue cargada con una muestra de semen diluido y las células fueron contadas en un microscopio óptico, con objetivo de 40X, el valor obtenido fue dado en millones $/ \mathrm{mL}$.

Prueba de Viabilidad. La viabilidad se evaluó con la coloración con Eosina/Nigrosina preparada según Barth \& Oko (1989). En un portaobjeto se preparo un frotis con una alícuota de $5 \mathrm{~mL}$ de la solución colorante y la misma cantidad de semen mezclado, después de secar al aire, la lámina fue analizada, contándose 100 células en microscopio óptico. Los resultados fueron presentados en porcentaje de espermatozoides viables (espermatozoides no coloreados de rosa). Los espermatozoides de color rosado (eosinófilos) se consideraron con membrana lesionada o muertos.

Análisis estructural de la membrana plasmática. Mediante el uso de una prueba hiposmótica (Hypo-osmotic swelling test) (Jeyendran et al., 1984) se analizó el estado estructural de la membrana plasmática ya que su integridad y buen funcionamiento es fundamental para el metabolismo espermático y para todas las etapas involucradas en el proceso de fertilización. Se preparó una solución de sacarosa 
CALVO, J.; ELHORDOY, D.; MODERNEL, A.; TAVARES, E.; ARAGUNDE, R.; ROHRER, V.; ROBERT, D.; VIQUEIRA, M. \& LOMBIDE, P. Obtención, evaluación y congelación de semen de antilope adulto (Addax nasomaculatus) del zoo-parque Lecocq de Montevideo-Uruguay. Int. J. Morphol., 34(1):276-279, 2016.

diluida en agua bidestilada (18 g/L) (Orozco Chávez et al., 2011). En un tubo Eppendorf se colocó $1 \mathrm{~mL}$ de la solución preparada y $100 \mu \mathrm{L}$ de semen para ser incubados a $37^{\circ} \mathrm{C}$ por 30 min. Posteriormente se cuentan cien espermatozoides y se consideran como viables aquellos cuyas colas están dobladas y/o cabezas agrandadas.

La termorresistencia espermática tiene por principio la exposición de los espermatozoides a las condiciones fisiológicas de temperatura del tracto genital femenino, a fin de estimar su capacidad de mantener la motilidad espermática. Se evaluó incubando las muestras por una hora en un baño maría a $38{ }^{\circ} \mathrm{C}$ (similar a la del tracto genital femenino) adaptando la técnica original (Dimitropulus, 1967) utilizada para la evaluación de la fertilidad bovina. La motilidad progresiva y el vigor espermático fueron evaluados inmediatamente después de realizada la dilución de la fracción espermática (tiempo 0), y a los 60 min (tiempo 1). La evaluación de la motilidad se realizó para las tres muestras de cada eyaculado, tomando una gota de cada dilución y colocándola sobre un portaobjeto temperado a $38^{\circ} \mathrm{C}$.

Congelado. El semen evaluado de cada macho y siempre refrigerado a $4{ }^{\circ} \mathrm{C}$ fue posteriormente diluido en diluyente Minitube ${ }^{\circledR}$ a una proporción de volumen final necesaria para envasar un mínimo de 50 x 106 espermatozoides móviles por pajuelas de $0,25 \mathrm{~mL}$.

Utilizando el método convencional, el semen refrigerado a $4{ }^{\circ} \mathrm{C}$ durante $2 \mathrm{~h}$ fue cuidadosamente envasado en pajuelas francesas de $0,25 \mathrm{~mL}$, a las que se les eliminó el excedente y sello con alcohol polivinilico en polvo. Las pajuelas fueron colocadas sobre una rejilla de metal en una caja de poliestireno con nitrógeno a una distancia de $6 \mathrm{~cm}$ de la superficie liquida durante 30 min de modo de ser enfriadas con los vapores de nitrógeno. Transcurrido este tiempo fueron sumergidas en el nitrógeno por 10 min para ser finalmente almacenadas dentro de los globest en el tanque de nitrógeno líquido a $-196^{\circ} \mathrm{C}$.
Descongelado. Transcurrido dos años, y con la finalidad de evaluar la viabilidad se procedió a retirar del tanque de nitrógeno una pajuela de cada macho y después de agitarla unos segundos al aire se sometieron a baño maría $\left(37^{\circ} \mathrm{C}\right)$ por $30 \mathrm{~s}$. Posteriormente fue retirada del agua, secada con papel, lavada con alcohol $70^{\circ}$ y cortada con tijera el extremo sellado por el operador y después el extremo con el algodón. El contenido de la pajuela es vertido a un tubo en baño a $37^{\circ} \mathrm{C}$. Posteriormente se toma una muestra y en lámina portaobjeto se contaron aproximadamente 100 espermatozoides en varios campos elegidos aleatoriamente. Fueron considerados como funcionalmente vivos (HOST+) los espermatozoides que presentaron edema, evidenciado por el enrollamiento de la cola y/o agrandamiento de la cabeza, ya que al estar sus membranas funcionales, estas permite la entrada del agua, lo que dobla o curva la cola en forma total o parcialmente. Aquellos con cola recta (HOST-) son considerados negativo por la incapacidad de su membrana para permitir el ingreso del agua.

\section{RESULTADOS}

Semen fresco. Los machos respondieron satisfactoriamente al método de sedación y extracción de semen. Se constato una variabilidad en los volúmenes de semen extraído. En relación al aspecto y color, se observó lechoso, pálido y ligeramente transparente en uno de los machos, mientras que en los otros dos se vio lechoso y amarillento. En la prueba de termorresistencia espermática, la motilidad progresiva, vigor espermático y viabilidad mostraron una disminución lineal según el tiempo de incubación en los tres machos en forma similar a lo que ocurre con otras especies de mamíferos. Las variables medidas en el análisis de semen fresco obtenido se expresan en las Tablas I, II y III.

Semen congelado. La prueba hipoosmótica (HOST) permitió constatar colas dobladas y/o cabezas agrandadas en un $35 \%$ en el macho 1, un $48 \%$ en el macho 2 y un $60 \%$ en el macho 3.

Tabla I. Volumen, color, motilidad de masa, motilidad individual y vigor del semen y espermatozoides de los tres antílopes adultos (Addax nasomaculatus) estudiados.

\begin{tabular}{lcccc}
\hline Macho & Volumen $(\mathbf{m L})$ & Aspecto y color & Motilida de masa & Motilidad individual y vigor \\
\hline 1 & 7 & Lechoso y pálido & $4 / 5$ & $80 \%-4 / 5$ \\
2 & 3,8 & Lechoso amarillento & $3 / 5$ & $50 \%-4 / 5$ \\
3 & 1,6 & Lechoso amarillento & $4 / 5$ & $80 \%-4 / 5$ \\
\hline
\end{tabular}

Tabla II. Concentración, número total de espermatozoides, porcentaje de viabilidad y porcentaje de anormalidades morfológicas de los espermatozoides en los antílopes adultos (Addax nasomaculatus) estudiados.

\begin{tabular}{lcccc}
\hline Macho & Concentración $\mathbf{( \mathbf { m m } ^ { 3 } )}$ & n total de zoides & Viabilidad $\mathbf{( \% )}$ & $\mathbf{( \% )}$ anormalidades \\
\hline 1 & 340000 & $2.380 \times 106$ & 70 & 50 (cabeza y cola) \\
2 & 240000 & $912 \times 10^{6}$ & 95 & 62 (cola, gota citoplasmatica) \\
3 & 730000 & $1168 \times 106$ & 80 & 45 (cabeza y cola) \\
\hline
\end{tabular}


Tabla III. Termoresistencia de los espermatozoides en los antílopes adultos (Addax nasomaculatus) estudiados.

\begin{tabular}{lccc}
\hline Macho & Tiempo 0 & Tiempo 1 (60 min) & Calificación \\
\hline 1 & $4(60 \%)$ & $2(45-50 \%)$ & Aceptable \\
2 & $3(50 \%)$ & $2(50 \%)$ & Aceptable \\
3 & $4(60 \%)$ & $2(45-50 \%)$ & Aceptable \\
\hline
\end{tabular}

\section{DISCUSIÓN}

Los animales respondieron satisfactoriamente a la manipulación y a la aplicación de un protocolo de sedación y extracción de semen estandarizado para otras especies domesticas domésticas de la familia bovidae.

Las diferencias de volumen de semen recogido en cada macho podemos decir que es similar a lo que ocurre en otros machos cuando se utiliza estimulación eléctrica.

En relación al uso de métodos y criterios de evaluación estandarizados para el espermiograma y congelación de semen de otras especies, los datos obtenidos se pueden considerar aceptables, validan la metodología utilizada y estimulan a continuar trabajando.

Debido a las diferencias bioquímicas y fisiológicas de los distintos componentes de los espermatozoides de mamíferos, es necesario poder determinar los efectos de la congelación y estandarizar los protocolos para esta especie. dada su condición de animales en cautiverio y en peligro de extinción. Nuestro laboratorio intenta, contribuir con este y otros ensayos al conocimiento para su preservación.

CALVO, J.; ELHORDOY, D.; MODERNEL, A.; TAVARES, E.; ARAGUNDE, R.; ROHRER, V.; ROBERT, D.; VIQUEIRA, M. \& LOMBIDE, P. Collection, evaluation and freezing of sperm antilope adult (Addax nasomaculatus) of the Lecocq zoo-park in Montevideo-Uruguay. Int. J. Morphol., 34(1):276-279, 2016.

SUMMARY: For almost 50 years sperm cryopreservation has been an important reproductive biotechnology that promotes the conservation of male germplasm. While today automated portable electronic systems have been developed, many technicians continue to use the conventional method of freezing for its practicality, low cost and efficiency. In this work we present preliminary data using standard protocols for assessing reproductive males of other species, about evaluation and freezing of adult antelope (Addax nasomaculatus) male gamete. Semen from three adult male antelope was obtained; we registered volume, color, motility, vigor, vitality, concentration and morphology. The semen was frozen by the conventional method and preserved under $-196^{\circ} \mathrm{C}$ nitrogen. Males responded satisfactorily to the method of sedation and extraction of semen. The evaluation of fresh and frozen semen showed acceptable preliminary data that validate the methodology and encourage them to continue working with this species because of their status of animals in captivity and in danger of extinction.

KEY WORDS: Sperm; Cryopreservation; Antelope.

\section{REFERENCIAS BIBLIOGRÁFICAS}

Amirat, L.; Tainturier, D.; Jeanneau, L.; Thotin, C.; Gérard, O.; Courtens, J. L. \& Anton, M. Bull semen in vitro fertility after cryopreservation using egg yolk LDL: a comparison with Optidyl, a commercial egg yolk extender. Theriogenology, 61(5):895-907, 2004.

Barth, A. D. \& Oko, R. J. Abnormal Morphology of Bovine Spermatozoa. Ames, Iowa State University Press, 1989.

de Souza Castelo, T.; Rodrigues Frota, T. \& Rodrigues Silva, A. Considerações sobre a criopreservação do sêmen de caprinos. Acta Vet. Bras., 2(3):67-7, 2008.

Dimitropulus, E. La signification du test de la thermoresistance dans l'application de la valeur fecondante du sperme congelé. Ann. Med. Vet., 4:215-24, 1967.

Forero Gonzalez, R. A. Efeito da criopreservação usando diferentes técnicas de congelação e crioprotetores sobre parámetros espermáticos e a integridade de membranas do espermatozoide bovino. Tesis doctoral. Pirassununga, Faculdade de Medicina Veterinária e Zootecnia, Universidade de São Paulo, 2004.

Januskauskas, A. \& Zilinskas, H. Bull semen evaluation post-thaw and relation of semen characteristics to bull's fertility. Vet. Med. Zoot, 17(39), 2002.

Jeyendran, R. S.; Van der Ven, H. H.; Perez-Pelaez, M.; Crabo, B. G. \& Zaneveld, L. J. Development of an assay to assess the functional integrity of the human sperm membrane and its relationship to other semen characteristics. J. Reprod. Fertil., 70(1):219-28, 1984.

Leizagoyen, C. Conservación "ex situ": los zoológicos y la genética de la conservación. Agrociencia, 9(1-2):597-602, 2005.

Moore, A. I.; Squires, E. L.; Bruemmer, J. E. \& Graham, J. K. Effect of cooling rate and cryoprotectant on the cryosurvival of equine spermatozoa. J. Equine Vet. Sci., 26(5):215-8, 2006.

Newby, J. \& Wacher, T. Addax nasomaculatus. The IUCN Red List of Threatened Species 2008:e.T512A13058429, 2008.

Orozco Chávez, V.; Rosemberg Barrón, M.; Santiani Acosta, A. \& Rodríguez Landeo, H. Evaluación de tres dilutores para la refrigeración de semen de caballo peruano de paso y sus efectos sobre la motilidad espermática e integridad funcional de la membrana. Cientifica., 8(2)104-14, 2011.

Direccion para Correspondencia:

Juan Calvo

Área Teriogenología

Facultad de Veterinaria

Universidad de la República

Alberto Lasplaces 1620

CP 11600

Montevideo

URUGUAY

Email: juancalvouy@gmail.com

Recibido : 05-08-2015

Aceptado: $10-11-2015$ 